\title{
Type-Shifting: Four Categories
}

\author{
Hamidreza Bahramian \\ Indiana University
}

\begin{abstract}
In formal semantics of natural languages, lexical items are considered to have types. For example, Euclid's Elements is of type Book or of type e (i.e. individuals). Whether a given type assignment is universal, that is whether the type assignment works in all felicitous contexts or not affords four possible answers. Either type assignments are universal or not, and then if they are, either they have a complex structure which accommodates all senses of the corresponding lexical items, or they are atomic types meaning that when the context changes, we have a different lexical entry. If the types are not universal, then the family of types assigned to a lexical item are either systematically related or not. If the notion that lexical items have types has an objective reality, then only one of these four possibilities is real. In this paper, we explore these four possibilities and examine some of the main approaches in the literature in view of these four categories. This categorization aims at giving a big picture of the issue of type-shifting, this time shaped around a different characterization; we want to see how the approaches are similar or different in their essential visions toward the phenomena of type-shifting.
\end{abstract}

Keywords: Euclid's Elements, Accommodates All Senses, Phenomena Of Type-Shifting, Lexical items, Hypothesis

\section{$1 \quad$ INTRODUCTION}

In type theoretical approaches to formal semantics of natural languages, a lexical item in a sentence is assigned a type and using a number of composition rules and based on the syntactic structure of the sentence, the types compose to converge to a single type called $t$, the semantic type for sentences, together with a statement specifying the conditions under which the sentence is true. For example consider the example (1-a) below

(1) a. Every linguist offended Fred.

b. Fred offended every linguist.

The syntactic structure of the sentence, its type assignments and the compositions are as follows(we omit the obvious truth conditions since it does not concern our discussion of type shifting):

(2)

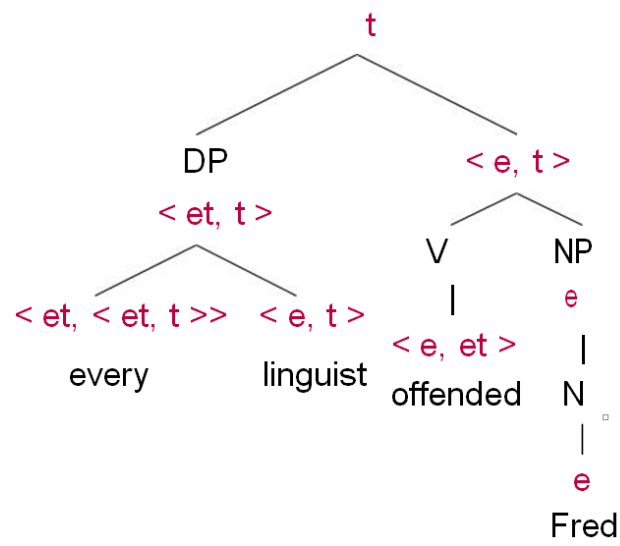

\section{Hamidreza Bahramian}

So the above type system correctly predicts that the example (1-a) is semantically interpretable. Consider the word every which has been assigned the type <et, ett>. We say the type <et, ett> is a locally correct type for the word every since this type assignment gives the expected result for a context, namely example (1-a). We call a type assignment, a globally correct assignment if the type assignment converges in all contexts containing the word. Whether there exist globally correct type assignments for lexical entries or not is a challenging question. There are at least four possibilities to be considered:

(Hypothesis A) Lexical items have globally correct type assignments. If the type assignment for a lexical item does not converge in a given interpretable context, then either the syntactic structure of the context needs to be changed or a new lexical entry needs to be defined.

(Hypothesis B) Some lexical items do not have globally correct type assignments but their locally correct assignments are systematically related. 
(3) (Hypothesis C) Some lexical items do not have globally correct type assignments and their locally correct assignments are not related.

(4) (Hypothesis D) Lexical items have globally correct type assignments. The type assignment for a lexical item may not converge in a given interpretable context, but the structure of types and the composition rules provide a mechanism that leads to semantic convergence.

Hypothesis B may seem not legitimately distinct from Hypothesis D. To illustrate the difference between the two, consider the following example: Assume people are lexical items and their names are type assignments and different countries are contexts. Hypothsis B assumes that for the case of some lexical items, there is no globally correct type assignment. So in our example, people have no names that can be used in all countries. Rather in each country they have different names, however the names for a given person are somehow related. So I am called Tao in China and Way in USA, but these two names are related, here one is the translation of the other in their respective languages. Hypothesis D on the contrary assumes that lexical items have globally correct type assignments. So in our example, people have a name that is used in all countries, for example I may be called Tao-Way-Facon-Weg. This name, however may undergo some transformations in each country, a transformation provided by the structure of the name which contains equivalent translations of some name. Hypothesis $\mathrm{C}$ will be dismissed quickly, yet it deserves to be mentioned because by contrast, it makes the other hypothesis more clear.

\section{HYPOTHESIS A}

(3)

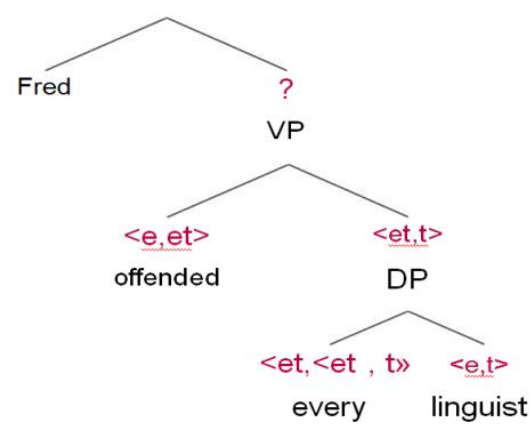

Consider the example (1-b). If the syntactic structure of the sentence is as the following then we cannot assign a type to $\mathrm{VP}$ as its children types are not reducible relative to the existing composition rules. According to hypothesis A, we can either maintain that here are actually two distinct words every ${ }_{1}$ and every ${ }_{2}$, each having a globally correct type assignment(one of them corresponding to the quantifier in subject position and the other to the quantifier in the object position) or to maintain that the syntactic structure of the sentence is the following rather than (3).

(4)

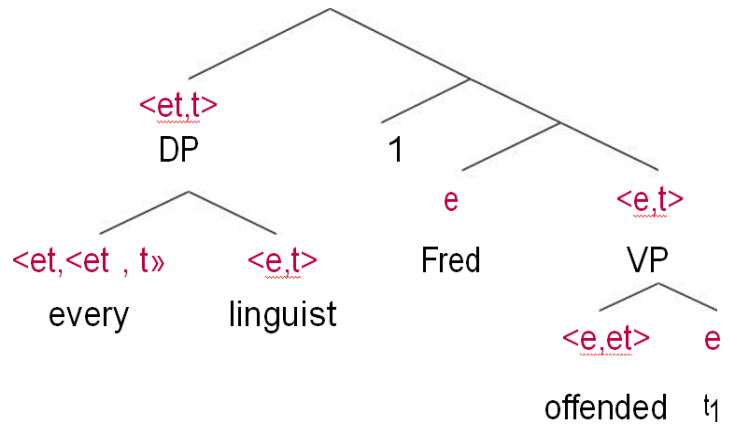

That is, it is postulated that the DP every linguist moves out of its place and adjoins to the S. This movement might affect the semantic of the sentence but it is not necessarily reflected in the phonetic form of the sentence. This proposal is based on a model of grammar like the inverted Y model of the Revised Extended Standard Theory and Government

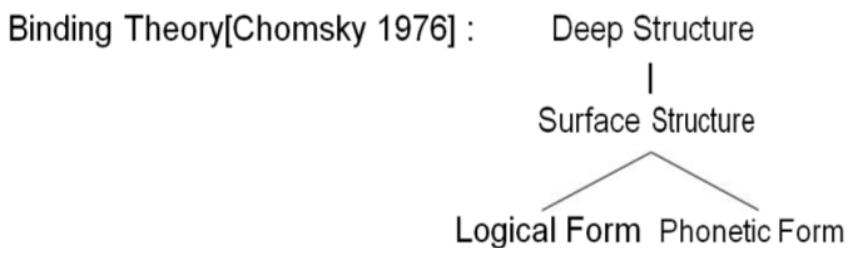


The model, however, is not enough to generate the structure in (4). So it is proposed([Kratzer and Heim 1998]) that traces must bear an index and the index on the trace has to be matched by an index on a variable binder somewhere. Now (4) with the following version of the predicate abstraction rule yields the desired interpretation for the example (1b).

(5) Predicate Abstraction Rule (PA): Let $\alpha$ be e branching node with daughters $\beta$ and $\gamma$, where $\beta$ dominates only a numerical index $\mathrm{i}$. Then for any variable assignment $\mathrm{a}, \mathrm{J} \alpha \mathrm{K}^{\mathrm{a}}=\lambda \mathrm{x} . \mathrm{J} \gamma \mathrm{K}^{\mathrm{a}(\mathrm{x} \rightarrow \mathrm{i})}$

So we will have

$\mathrm{J} 1\left[\right.$ Fred [offended $\left.\left.\mathrm{t}_{1}\right]\right] \mathrm{K}=\lambda \mathrm{x}$. Fred offended $\mathrm{x}$

4. Hamidreza Bahramian and therefore $\mathrm{J}(4) \mathrm{K}=1$ iff for every $\mathrm{x}$ such that $\mathrm{x}$ is a linguist, Fred offended $\mathrm{x}$.

So using movement of quantifier we can avoid type ambiguity. It has been argued that the choice of movement is not just a trick to get ride of type shifting, rather it has advantages in dealing with three phenomenas: scope ambiguity, antecedent-contained deletion and bound-variable phenomena[Kratzer and Heim 1998].

\section{HYPOTHESIS B}

The approach of type shifting can be formulated with a considerable systematicity. That is, for the words that have multiple types, the types are systematically related to each other in the sense that given an object of one type we can construct an object of the other ones. For example, in the following sentence

(6) Fred and every linguist arrived. if Fred is to be considered to have type e and every linguist to have type <et, $\mathrm{t}>$, then hypothesis $\mathrm{B}$ maintains that at least one of them has to have more than one type and the types have to be related systematically ${ }^{1}$. One example of applying this hypothesis, is developed in [Partee 1986], where common nous are assigned a family of three different types along with mappings between the types. It is a departure from Montague's framework, where there is a one-to-one correspondence between syntactic categories and semantic types, but it still retains a kind of correspondence between the two, only this time a one-to-many correspondence. She provides evidences showing that there exist referring, predicative and quantificational uses of NP's. As for referential interpretation, the evidence is the fact that although any singular NP can bind a singular pronoun in its domain, only some NP's can bind a discourse anaphora:

Fred/the man/a man walked in. He looked tired.

Every man/no man/more than one man walked in. *He looked tired.

shows that some NP's have a referential interpretation while (8) shows that not all NP's have this interpretation.

The evidence for predicative interpretation of some NP's comes from the fact that they are conjoinable with adjective phrases (that is, with something of type $<e, t>$ ):

Ann considers Fred competent in semantics and an authority on unicorns.

Now if we maintain that conjunction requires identical types, then these NP's need no have a predicative interpretation as there is no evidence (according to [Partee 1986]) to assume that adjective phrases can have quantificational of referential interpretations. So [Partee 1986] maintains that all NPs have meanings of type <et, $t$, while only some of them have meanings of types e and $\langle e, t\rangle$. She then proposes some systematic mappings between the types as it is shown in Figure 1. In the figure, the circles represent the types and the arrows represent the functions which map objects of one type into corresponding objects in the other type. In general, these mappings are not considered to be universal, except for those maps that are derivable directly from the type theory.

1 By contrast, hypothesis A would maintain that the word every actually has two different lexical entries, one of type <et, <et, $t$ » and the other of type <et, e>. It is then the latter entry which makes the sentence (6) interpretable.

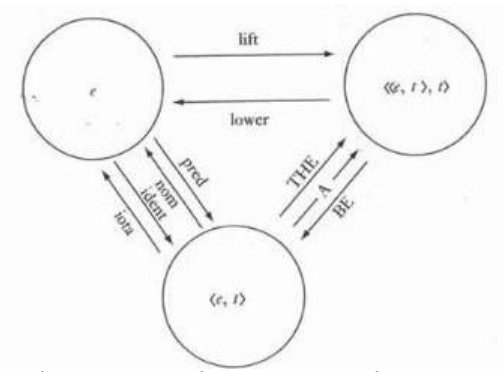

Fig. 1. Mappings between the three types that NPs can have meanings of.[Partee 1986] 
The mappings are defined as below where the first five functions are total and the remaining two are partial functions:

$$
\begin{aligned}
& \text { (1)ident }(y)=\lambda x \cdot(x=y) \\
& \text { (2) } \operatorname{lift}(\mathrm{x})=\lambda \mathrm{f} \text {.(f } \mathrm{x}) \\
& \text { (3) } \mathrm{THE}(\mathrm{f})=\lambda \text { д. } \exists \mathrm{x}(\forall \mathrm{y}((\mathrm{f} \mathrm{y}) \leftrightarrow(\mathrm{x}=\mathrm{y})) \wedge(\text { ду })) \\
& (4) \mathrm{a}(\mathrm{f})=\lambda д . \exists \mathrm{x}((\mathrm{f} \mathrm{x}) \wedge(\text { дx })) \\
& (5) \mathrm{BE}(\mathrm{P})=\lambda \mathrm{x}(\mathrm{P}(\lambda \mathrm{y} \cdot(\mathrm{y}=\mathrm{x}))) \\
& \text { (6) } \operatorname{iota}(f)=\text { the unique } d \in D_{e} \text { such that } f(d) \text { is true } \\
& \text { e to }<\mathrm{e}, \mathrm{t}> \\
& \text { e to }<\text { et, } t> \\
& \text { (7) lower }(\mathrm{P})=\text { the unique } \mathrm{d} \in \mathrm{D}_{\mathrm{e}} \text { such that for all } \mathrm{f} \in \mathrm{D}_{<\mathrm{e}, \mathrm{t}}>, \mathrm{P}(\mathrm{f}) \text { is true if and only if } \mathrm{f}(\mathrm{d}) \text { is true }<\text { et, } \mathrm{t}>\text { to } \mathrm{e}
\end{aligned}
$$

Another possibility within hypothesis B, is the introduction of type-lifting rules to the semantic system. [?] propose a system in which each syntactic category is assigned a specified type, considered as its simplest type, and a set of typelifting rules is defined which define all the types associated with each syntactic category. In this approach, whenever there is a type mismatch in the process of computing the semantic interpretation of a given sentence, we first try the simplest type associated with the category of the word under consideration, if it does not work, we find the lowest type possible using the type-lifting rules. For example consider the category of proper nouns. The simplest type assigned to this category is the type e and the type-lifting rule for this category is as follows:

(10) Let $\alpha$ be a term and let $\alpha$ ' be its denotation (which is an object of type e). Then $\alpha$ also has a denotation $\alpha$ " of type <et, $\mathrm{t}>$, where $\alpha^{\prime \prime}=\lambda \mathrm{P}\left[\mathrm{P}\left(\alpha^{\prime}\right)\right]$ ( $\mathrm{P}$ is a variable of type $<\mathrm{e}, \mathrm{t}>$ ). Similarly for other syntactic categories we define specific simplest types and type-lifting rules. [Partee 1986] shows that although the framework predicts readings over a wide range of straightforward cases, still there are readings which this framework is not able to predict. Another variant of the hypothesis B is Generative Lexicon framework. [Pustejovsky 2006] argues that lexical information and the representations used in composing meaning is not separable from our commonsense knowledge. Therefore he introduces a knowledge representation framework that can represent the sense(s) of the word along with some of its inferential relations. In this framework the lexical structure of lexical item consists of four levels:

- $\quad$ Lexical typing structure

- $\quad$ Argument structure: specifying the number and nature of the arguments to a predicate

6 Hamidreza Bahramian

- $\quad$ Event structure: defining the event type of the expression

- Qualia structure

Quailia structure represents the modes of explanation and consists of the following modes:

- $\quad$ Formal: the basic category of the word

- $\quad$ Constitutive: the relation between an object and its constituent parts

- $\quad$ Telic: the purpose or function of the object

- Agentive: the origin of the object or the factors contributing to it

Figure 2 gives a visual representation of the knowledge representation framework that was defined above.

$$
\left[\begin{array}{l}
\alpha \\
\text { ARGSTR }=\left[\begin{array}{l}
\text { ARG1 }=x \\
\cdots
\end{array}\right] \\
\text { EVENTSTR }=\left[\begin{array}{l}
\mathrm{E} 1=e_{1} \\
\cdots
\end{array}\right] \\
\text { QUALIA }=\left[\begin{array}{l}
\text { CONST }=\text { what } x \text { is made of } \\
\text { FORMAL }=\text { what } x \text { is } \\
\text { TELIC }=\text { function of } x \\
\text { AGENTIVE }=\text { how } x \text { came into being }
\end{array}\right]
\end{array}\right]
$$

Fig. 2. Lexical structure for $\alpha$

In this framework each lexical item has a single knowledge representation which includes all the aspects of the item meaning. When the lexical item is embedded in a context, "mutually compatible roles in the lexical decompositions of each word become more prominent.'2.

To demonstrate how type-shifting is handled in this framework, consider the following example:
a. Ann wants Fred to leave.
b. Ann wants to leave.
c. Ann wants a beer. 
Now want is assumed to have a proposition as an argument. So the arguments to leave and a beer are coerced into a propositional type. [Pustejovsky 1995] proposes the following rule to convert the type of the complement to the verb where it is needed:

Type Coercion: a semantic operation that converts an argument to the type which is expected by a function, where it would otherwise result in a type error.

One case of coercion is subtype coercion. Consider the sentence in the following example:

$$
\text { Fred drives a Honda to work. }
$$

Assuming the type selected type by the verb in (13) is vehicle, then we need the following subtyping relation: Honda $\leq$ car $\leq$ vehicle, where $\mathrm{a} \leq \mathrm{b}$ means type $\mathrm{a}$ is a subtype of type $\mathrm{b}$. So by type coercion we get the expected semantic interpretation for (13). In general [Pustejovsky 1995] defines a sybtyping coercion relation, $\Theta$ as follows:

2[Pustejovsky 2006]

$$
\frac{\alpha: \mathrm{T} 1, \Theta\left[\mathrm{T}_{1} \leq \mathrm{T} 2\right]: \mathrm{T} 1 \rightarrow \mathrm{T} 2}{\Theta[\mathrm{T} 1 \leq \mathrm{T} 2](\alpha): \mathrm{T} 2}
$$

Which means that, given an expression $\alpha$ of type $\tau_{1}$ which is a subtype of $\tau_{2}$, there is a coercion from $\tau_{1}$ to $\tau_{2}$ which changes the type of $\alpha$ from $\tau_{!}$to $\tau_{2}$.

Another case of coercion is complement coercion. Consider the following example:
a. Fred began a book.
b. Fred began reading a book.
c. Fred began to read a book.

In the lexical structure associated with the verb begin, the type of the second argument is defined as an event, that is of type event. Where an object of that type is not directly given to the verb, as in (15-b) or (15-c), a coercion is applied to convert the object to the appropriate type.But the coercion is successful only if the NP under conversion allows such a conversion. That is, if in the lexical structure of the NP, this possibility has been defined. Sentence (15-a) is a more complicated case because book has a relation with event only though its informational aspect. For words like book which refer to two or more aspects of an object's meaning, [Pustejovsky 1995] proposes a complex type structure, namely dotted type, defined as follows:

$$
\frac{\alpha: \mathrm{T} 1 \alpha: \mathrm{T}_{2}}{\operatorname{lcp}(\alpha): \mathrm{T} 1 . \mathrm{T} 2}
$$

where lcp is the type constructor and $\tau_{1}, \tau_{2}$ are some senses for $\alpha$. (16) says that two types are able to combine to form a complex type. Now the lexical structure of book, shown in Figure 3 below, is defined as a dot object, constructed out of the types information and physobj, informational and physical types respectivley.

$$
\left[\begin{array}{l}
\text { book } \\
\text { ARGSTR }=\left[\begin{array}{l}
\text { ARG1 }=y \text { :information } \\
\text { ARG2 }=x: \text { phys_obj }
\end{array}\right] \\
\text { QUALIA }=\left[\begin{array}{l}
\text { FORM }=\text { hold }(\mathrm{x}, \mathrm{y}) \\
\text { TELIC }=\text { read }(e, w, x \cdot y) \\
\text { AGENT }=\text { write }\left(e^{\prime}, \mathrm{v}, \mathrm{x} \cdot \mathrm{y}\right)
\end{array}\right]
\end{array}\right]
$$

Fig. 3. Lexical structure for book

Now because the NP a book does not satisfy the type required by the predicate begin, the verb, using the qualia structure of book, coerces the NP to an object of type of event. Now consider a more challenging situation:

(17) Fred believes the book.

The expected type of the book is < et, $\mathrm{t}>$ since it is a generalized quantifier, but in the sentence (17), it assumes the interpretation of a proposition. To get this type, we first use an operator on the dot type info.physobj, called pumping operator, to get an object of type info and then since in $\mathrm{fo} \leq$ prop, we will get an object of type prop:

8 Hamidreza Bahramian

$$
\frac{\Sigma_{1}(\text { in } \mathrm{f} \text { o.physobj }): \text { in } \mathrm{fo}, \Theta[\text { in } \mathrm{f} \mathrm{o} \leq \text { prop }]: \text { in } \mathrm{fo} \rightarrow \text { prop }}{\Theta[\text { in } \mathrm{fo} \leq \operatorname{prop}]\left(\Sigma_{1}(\text { in } \mathrm{f} \text { o.physobj })\right): \text { prop }}
$$


Pustejovsky's theory have been criticized for being based on the assumption that the meaning of a linguistic expression is constituted by at least some of its inferential relations (this assumption is called inferential role semantics, IRS for short)[Fodor and Lepore 1998]. Instead of IRS axiom, they advocate the null hypothesis, which asserts that the only thing a lexical entry specifies is the denotation of the item it describes. In Pustejovsky's theory, the complexity of lexical entries is argued to be required for at least two reasons: First, lexical generatively cannot be explained if lexical entries do not provide enough complex data. Second, to specify how words are semantically related, a rich lexical semantics is required. [Fodor and Lepore 1998] argue that lexical ambiguity is compatible with a denotational semantics (I think hypothesis A would be a compatible solution) and that Inter-lexical semantic relations presupposes that the notion of analyticity (i.e. lexical entailment) is viable whereas it is argued to the contrary. So in short, it is argued that there is nothing in Pustejovsky's theory that proves there is something more to lexical representations than denotations.

Knowledge representation framework is only one possibility within hypothesis D. There can be other frameworks which exhibit the same functionality. One such framework is discussed in [Piñango and Deo 2015]. Like Pustejovsky's theory, this approach also relies on a rich lexical structure as it requires that lexical entries for each expression to include "information about the possible dimensions along which entities may be construed as structured individuals e.g. the spatial, temporal, informational dimension of entities, or any intrinsic or conventionalized ordering of their partstructure". It differs from Generative Lexicon, in that first, it only applies to aspectual verbs and second, aspectual verbs select for structured individuals and the provider of the desired interpretation is a constrained set of homomorphic functions (that is the functions mapping individuals to their (axial) extents in different dimensions) rather than coercion. Here we give a brief account of the framework proposed in [Piñango and Deo 2015]. It has been assumed that the complements to the verbs like begin and enjoy must be eventive. [Piñango and Deo 2015]. For example in the sentence (19) where the book denotes an individual,

(19) Fred began the book. the typical interpretation is that it means some events involving the book (for example, reading or writing) is oc-curring. This phenomena, which has been called complement coercion, has a process-based account as the following: "type-mismatch triggered by failure of the complement to meet the selectional restrictions of the verb, subsequent failure of straightforward compositionality, and the repair of the mismatch via type-shifting" 3 .

Since coercion phenomena has no overt morpho-syntactic reflexes, that is the change in typing is occurring on the purely semantic plane, it therefore can be used to study purely semantic processes, hence the neuro/psycholinguistic interest in this phenomena. so a number of experimental studies have been carried out to study the neuro- and psycholinguistic semantic modelling along with lexical semantic analyses. The result of these studies show that there is a computational cost associated with the interpretation of sentences that require complement coercion. This finding initially led to the idea that the coercion effect has a unified description. [Katsika et al. 2012], however, argue that in the previous studies the semantic differences between verbs have been neglected, showing that there is a difference in processing aspectual verbs versus psychological verbs: only aspectual verbs (e.g. begin, finish) exhibit cost, not psychological verbs (e.g. enjoy, savor). They argue that aspectual verbs, in general, do not select event-denoting complements. They present the following counterexample:

a. This is the famous perch that officially begins the Appalachian Trail.

b. A little porcelain pot finished the row.

c. Defoe (1661-1731) begins the list of writers of the period of people's influence...

d. "Under The Sun" ends the album with the s-1-o-o-o-o-o-o-w-est Sab riff ever..

[Katsika et al. 2012] argue that there is no eventive complement (syntactically explicit or coerced) in these examples and conclude that "the interpretations of sentences containing aspectual verbs systematically make reference to parthood relations between objects along a range of familiar (and, sometimes not so familiar) dimensions".

[Katsika et al. 2012] propose that individuals are structured, in the sense that they relate to a one dimensional directed path (definition of which is given below) along a dimension determined by the context. The interpretation of aspectual verbs, then, "is dependent on determining the specific dimension along which the complement denotation is structured".

So instead of the process of type-mismatch and coercion-based type-shifting, here we have the process of determination of the dimension from the set of possibilities that the subject and complement denotations and their interaction entail. The increased processing cost which the aspectual verbs exhibit is then explained by the cost of the latter process.

3.0.1 One-dimensional directed paths. [Krifka 1998] defines the notion of one-dimensional directed path as follows: A join semi-lattice is a tuple $\langle U,+>$ where $U$ is a set of entities and + is the two-place sum operation. For any two elements $x, y \in U$, their sum (join) $x+y \in U$. A part structure $P$ is a join semi-lattice $\langle U,+>$ whose elements satisfy

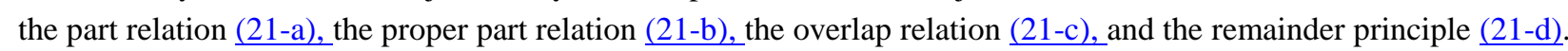




$$
\begin{aligned}
& \text { a. } \forall \mathrm{x}, \mathrm{y} \in \mathrm{U}[\mathrm{x} \leq \mathrm{y} \rightarrow \mathrm{x}+\mathrm{y}=\mathrm{y}] \\
& \text { b. } \forall \mathrm{x}, \mathrm{y} \in \mathrm{U}[\mathrm{x}<\mathrm{y} \text { iff } \mathrm{x} \leq \mathrm{y} \& \mathrm{x}+\mathrm{y}, \mathrm{y}] \\
& \text { c. } \forall \mathrm{x}, \mathrm{y} \in \mathrm{U}[\mathrm{x}+\mathrm{y} \text { if } f \exists \mathrm{z} \in \mathrm{U}[\mathrm{z} \leq \mathrm{x} \& \mathrm{z} \leq \mathrm{y}]] \\
& \text { d. } \forall \mathrm{x}, \mathrm{y} \in \mathrm{U}[\mathrm{x}<\mathrm{y} \rightarrow \exists ! \mathrm{z}[\neg[\mathrm{z}+\mathrm{x}] \& \mathrm{x}+\mathrm{z}=\mathrm{y}]]
\end{aligned}
$$

A one-dimensional directed path structure (dps for short) D is a part structure that is convex and linear and whose adjacent parts are totally ordered by the precedence relation. By adjacent parts, we mean elements of a part-structure that are externally connected and non-overlapping and by precedency relation (denoted as $\prec$ ) we mean the relation satisfying the following conditions:

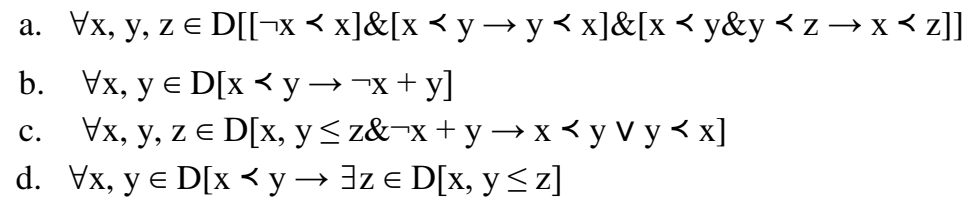

and finally by totally ordered we mean that the structure satisfies the following:

$$
\forall x, y \in D[\neg x+y \rightarrow x \prec y \vee y \prec x]
$$

[Krifka 1998] gives the examples of the text of a book as a dps, assuming the chapters of a book to be the adjacent parts of the book and $\prec$ to be the order of these chapters relative to each other.

\section{Hamidreza Bahramian}

Now [Katsika et al. 2012] assume four mereological domains in the ontology: D , the domain of ordinary individuals, and $\mathrm{E}$, the domain of event individuals, $\mathrm{S}$, the domain of spatial intervals and I the domain of temporal intervals I. The first three are part structures and the temporal interval is a dps. They are partial functions that map entities from each of the four domains to the other domain. They then define the notion of structured individual as

(24) An individual $\mathrm{x}$ of any type $\tau$ is taken to be a structured individual relative to a function $\mathrm{f}$ of any type $(\tau, \sigma)$ if $\mathrm{f}(\mathrm{x})$ is an axis and $\mathrm{f}$ is a homomorphism from the part structure of $\mathrm{x}$ to the axis $\mathrm{f}(\mathrm{x})$

Having all the machinery ready, aspectual verbs are taken to select for structured individuals and this selectional requirement is defined as a lexical presupposition. Now the meaning of begin can be defined as To demonstrate how this framework is used consider the following example:

(25)

$\operatorname{Begin}(\mathrm{x})(\mathrm{y})$ is defined iff $\mathrm{x}$ is a structured individual with respect to the contextually determined function $f_{c}$. If defined, begin $(x)(y)$ is true iff there is some function $f^{\prime}$ (possibly identical to $f$ ) such that $f^{\prime}(y)$ is a small initial subpart of the axis $f_{c}(x)$.

where $f_{c}$ is a function from a constrained set of functions that are lexically listed as part of the general meaning for this class of verbs. The choice of $\mathrm{f}_{\mathrm{c}}$ is determined by the context.

To demonstrate how this framework is used consider the following example:
a. This famous perch begins the Appalachian Trail.
b. $J$ beginK $=\lambda x_{\tau} \lambda y_{\sigma}:$ struct $-\operatorname{ind}_{\mathrm{fc}}(\mathrm{x}) \cdot \exists \mathrm{f}^{\prime}\left[\mathrm{f}^{\prime}(\mathrm{y})<_{\text {smal } 1-\text { init }} \mathrm{f}_{\mathrm{c}}(\mathrm{x})\right]$
c. $\mathrm{J}$ this famous perchK $=\mathrm{p}$
d. $\mathrm{J}$ the Appalachian TrailK $=\mathrm{a}$

so (26-a) is defined if and only if a is a structured individual which has a spatial dimension. If defined, (26-a) is true if and only if there is a function $\mathrm{f}^{\prime}$ mapping $\mathrm{p}$ to the initial part of the spatial extension of a.

\section{HYPOTHESIS C}

This hypothesis states that some lexical items do not have globally correct type assignments and their locally correct assignments are not related. If there is a lexical item that has semantic types which are unrelated then, unless the unrelated types correspond to unrelated semantic meanings, it seems reasonable to assume that this is a defect of the semantic system under consideration. This hypothesis either entails that the semantic system is something beyond the force of systematization or it is something chaotic. The latter case contradicts our cognitive abilities regarding semantics and the former endorses the limited nature of any systematization of semantic systems. 


\section{HYPOTHESIS D}

The last possibility is to maintain that lexical items do have globally correct types, however types need not be atomic ones rather they can be a complex sum of a set of types. Whenever there is a type mismatch in computing the semantic value of a felicitous sentence, the type of the lexical item that causes the mismatch, through its structure, provides objects of the required types:

\section{(27) Fred Mastered Euclid's Elements.}

Assuming Euclid's Elements is an object of type Book and master is the dependent type Informational $\rightarrow$ Human $\rightarrow$ Universe $^{4}$, the computation for (27) will fail. [Bahramian and Nematollahi 2017] propose a mathematical model, using identity types in pure intentional type theory, in which an object of a type can provide objects of related types through the structure of its type. For example the structure of the type Book is defined as:

$($ Book Prototype $=$ Physical Prototype $)=($ Book Prototype $=$ Informational Prototype $)$

The type Informational is defined as

$$
\text { (Informational Prototype = Informational Prototype) }
$$

and the type Physical is defined as

$$
\text { (Physical Prototype = Physical Prototype) }
$$

Now the physical object corresponding to the book object $b$, calling it $b$ qua physical, is given as the following:

(28) b qua physical $=b_{p}^{-1} \circ f\left(b_{p}\right) \circ b_{i}^{-1} \circ f^{-1}\left(b_{i}\right)$.

where $\mathrm{f}$ is a function from (Book Prototype = Physical Prototype) to (Book Prototype = Informational Prototype), induced by the object $b$ ([Univalent Foundations Program 2013] Lemma 2.10.1), and $b_{p}$ and $b_{i}$ are elements of types $($ Book Prototype $=$ Physical Prototype $)$ and (Book Prototype $=$ Informational Prototype $)$ respectively.

The informational object corresponding to the book object b, calling it b qua Informational, is given as the following:

$$
\mathrm{b} \text { qua Informational }=\mathrm{b}_{\mathrm{i}}^{-1} \circ \mathrm{f}^{-1}\left(\mathrm{~b}_{\mathrm{i}}\right) \circ \mathrm{b}_{\mathrm{p}}^{-1} \circ \mathrm{f}\left(\mathrm{b}_{\mathrm{p}}\right) \text {. }
$$

Note that the components that constitute the structure of $b$ qua Physical and b qua Informational are the same, only the order of the composition is different. In other words, b qua Physical and b qua Informational refer to the same thing, each only accentuate a different aspect of the thing.

So instead of shifting the types of Euclid's Elements or master, Euclid's Elements, being an object of type Book, provides an object of type Informational; an object, given not by a map between types, but implied as a consequence of the structure of the type Book.

\section{REFERENCES}

[1]. Hamidreza Bahramian and Narges Nematollahi. 2017. Copredication in homotopy type theory. (2017).

[2]. N. Chomsky. 1976. Conditions on Rules of Grammar. https://books.google.com/books?id=vbkcHQAACAAJ

[3]. Jerry A Fodor and Ernie Lepore. 1998. The emptiness of the lexicon: reflections on James Pustejovsky's The Generative Lexicon. Linguistic Inquiry 29, 2 (1998), 269-288.

[4]. Argyro Katsika, David Braze, Ashwini Deo, and Maria Mercedes Piñango. 2012. Complement coercion: Distinguishing between type-shifting and pragmatic inferencing. The mental lexicon 7, 1 (2012), 58-76.

[5]. Angelika Kratzer and Irene Heim. 1998. Semantics in generative grammar. Blackwell.

[6]. Manfred Krifka. 1998. The origins of telicity. Events and grammar 197235 (1998).

[7]. Barbara Partee. 1986. Noun phrase interpretation and type-shifting principles. (1986).

[8]. Maria Mercedes Piñango and Ashwini Deo. 2015. Reanalyzing the complement coercion effect through a generalized lexical semantics for aspectual verbs.

[9]. Journal of Semantics 33, 2 (2015), 359-408.

[10]. James Pustejovsky. 1995. The Generative Lexicon. The MIT Press.

[11]. 12Hamidreza Bahramian James Pustejovsky. 2006. Introduction to Generative Lexicon. Unpublished manuscript. Microsoft Word file (2006).

[12]. Univalent Foundations Program. 2013.

[13]. Homotopy Type Theory: Univalent Foundations of Mathematics. http://homotopytypetheory.org/book, Institute for Advanced Study. 